\title{
Event-by-Event Fluctuations of Particle Ratios in Heavy-Ion Collisions
}

\author{
A. Tawfik* \\ Hiroshima University, 1-7-1 Kagami-yama, Higashi-Hiroshima Japan
}

\begin{abstract}
We study event-by-event dynamical fluctuations of various particle ratios at different energies. We assume that particle production in final state is due to chemical equilibrium processes. We compare results from resonance gas model with available experimental data. At SPS energies, the model can very well reproduce the experimentally measured fluctuations. We make predictions for dynamical fluctuations of strangeness and non-strangeness particle ratios. We found that the energy-dependence is non-monotonic. Furthermore, we found that fluctuations strongly depend on particle ratios.
\end{abstract}

\section{Introduction}

Understanding dynamical properties of hot and dense matter is a key question in heavy-ion collisions experiments. Event-by-event fluctuations have been suggested [1, 2, 3, to provide comprehensive characteristics of this matter. They are crucial observations to check hypothesis of chemical equilibrium in heavy-ion collisions [4. Event-by-event fluctuations of very few particle ratios have been studied in several experiments at SPS and RHIC energies [5, 6, 7. In this letter, we purpose to study energy-dependence of event-by-event fluctuations in hadron resonance gas model. Hypothetical phase transition to and from quark-gluon plasma can be characterized by large fluctuations in particle yields 1, 2, 3, which are accompanied by volume fluctuations. The latter can be eliminated, when particle ratios are considered [3]. In this letter, we try to give answers to the questions, whether strangeness quarks enhance dynamical fluctuations and whether critical endpoint can be localized by means of dynamical fluctuations. Since experimental measurements of event-by-event fluctuations are very much limited, we hope that our predictions encourage specifying certain particle ratios and measure their dynamical fluctuations. In this letter, we make predictions for dynamical and statistical fluctuations of different particle ratios in dependence on energy. The values of fluctuations strongly depend on particle ratios. In some particle ratios, dynamical fluctuations are smaller than statistical ones. In other particle ratios, dynamical fluctuations are slightly greater than statistical ones. We also found that fluctuations of hybrid and cascade baryons are dominant. All these predictions are phenomenologically of great interest.

\section{Model}

Pressure in hadronic phase is given by contributions from all hadron resonances treated as a free gas [8, 9, 10, 11. The resulting pressure accounts for free as well as for strong interactions between resonances. In previous work [11, we have proved that the thermodynamics of strongly interacting system of hadron resonances can be approximated by an ideal gas of stable and resonance hadrons.

At finite temperature $T$, strangeness $\mu_{S}$ and iso-spin $\mu_{I_{3}}$ and baryo-chemical potential $\mu_{B}$, pressure of one hadron reads

$$
p\left(T, \mu_{B}, \mu_{S}, \mu_{I_{3}}\right)=\frac{g}{2 \pi^{2}} T \int_{0}^{\infty} k^{2} d k \ln \left[1 \pm \gamma \lambda_{B} \lambda_{S} \lambda_{I_{3}} e^{\frac{-\varepsilon(k)}{T}}\right],
$$

*tawfik@physik.uni-bielefeld.de 
where $\varepsilon(k)=\left(k^{2}+m^{2}\right)^{1 / 2}$ is single-particle energy and \pm stands for bosons and fermions, respectively. $g$ is spin-isospin degeneracy factor. $\gamma \equiv \gamma_{q}^{n} \gamma_{s}^{m}$ are quark phase space occupancy parameters, where $n$ and $m$ being number of light and strange quarks, respectively. In this letter, we explicitly use the equilibrium value for $\gamma$, the unity. $\lambda=\exp (\mu / T)$ is fugacity, where $\mu$ is chemical potential multiplied by corresponding charge.

Quark chemistry is given by relating hadronic chemical potentials to quark constituents. $\mu_{B}=$ $3 \mu_{q}$ and $\mu_{S}=\mu_{q}-\mu_{s}$, where $q$ and $s$ being light and strange quark quantum number, respectively. The baryo-chemical potential for light quarks is $\mu_{q}=\left(\mu_{u}+\mu_{d}\right) / 2 . \mu_{S}$ is calculated as a function of $T$ and $\mu_{B}$ under the condition of strangeness conservation. Iso-spin chemical potential $\mu_{I_{3}}=$ $\left(\mu_{u}-\mu_{d}\right) / 2$.

Particle number density is given by derivative of partition function in Eq. 1 with respect to the chemical potential of interest. Fluctuations in particle number are given by susceptibility density, which is second derivative with respect to chemical potential.

$$
\begin{aligned}
\langle n\rangle & =\sum_{i} \frac{g_{i}}{2 \pi^{2}} \int d k k^{2} \frac{e^{\left(\mu_{i}-\varepsilon_{i}\right) / T}}{1 \pm e^{\left(\mu_{i}-\varepsilon_{i}\right) / T}} \\
\left\langle(\Delta n)^{2}\right\rangle & =\sum_{i} \frac{g_{i}}{2 \pi^{2}} \int d k k^{2} \frac{e^{\left(\varepsilon_{i}-\mu_{i}\right) / T}}{\left(e^{\left(\varepsilon_{i}-\mu_{i}\right) / T} \pm 1\right)^{2}}=\sum_{i} \frac{g_{i}}{2 \pi^{2}} \int d k k^{2} \frac{\left\langle n_{i}\right\rangle}{1 \pm e^{\left(\mu_{i}-\varepsilon_{i}\right) / T}}
\end{aligned}
$$

After freeze out, hadron resonances decay either to stable particles or to other resonances. Particle number and fluctuation density in final state have to take into account this chemical process.

$$
\begin{aligned}
\left\langle n_{i}^{\text {final }}\right\rangle & =\left\langle n_{i}^{\text {direct }}\right\rangle+\sum_{j \neq i} b_{j \rightarrow i}\left\langle n_{j}\right\rangle, \\
\left\langle\left(\Delta n_{j \rightarrow i}\right)^{2}\right\rangle & =b_{j \rightarrow i}\left(1-b_{j \rightarrow i}\right)\left\langle n_{j}\right\rangle+b_{j \rightarrow i}^{2}\left\langle\left(\Delta n_{j}\right)^{2}\right\rangle
\end{aligned}
$$

where $b_{j \rightarrow i}$ being branching ratio for decay of $j$-th resonance to $i$-th particle. Chemical freeze out is characterized by $s / T^{3}$ [12], where $s$ is the entropy density.

Fluctuations of particle ratio $n_{1} / n_{2}$ are 3

$$
\sigma_{n_{1} / n_{2}}^{2}=\frac{\left\langle\left(\Delta n_{1}\right)^{2}\right\rangle}{\left\langle n_{1}\right\rangle^{2}}+\frac{\left\langle\left(\Delta n_{2}\right)^{2}\right\rangle}{\left\langle n_{2}\right\rangle^{2}}-2 \frac{\left\langle\Delta n_{1} \Delta n_{2}\right\rangle}{\left\langle n_{1}\right\rangle\left\langle n_{2}\right\rangle}
$$

which include dynamical as well as statistical fluctuations. Third term of Eq. 6 counts for fluctuations from hadron resonances that decay into particle 1 and particle 2, simultaneously. In such a mixing channel, all correlations including quantum statistics ones are taken into account. Obviously, this decay channel results in strong correlated particles. To extract statistical fluctuation, we apply Poisson scaling in mixed decay channels ${ }^{1}$,

$$
\left(\sigma_{n_{1} / n_{2}}^{2}\right)_{s t a t}=\frac{1}{\left\langle n_{1}\right\rangle}+\frac{1}{\left\langle n_{2}\right\rangle}
$$

Subtracting Eq. [7from Eq. [6] we get dynamical fluctuations of particle ratio $n_{1} / n_{2}$.

$$
\left(\sigma_{n_{1} / n_{2}}^{2}\right)_{d y n}=\frac{\left\langle n_{1}^{2}\right\rangle}{\left\langle n_{1}\right\rangle^{2}}+\frac{\left\langle n_{2}^{2}\right\rangle}{\left\langle n_{2}\right\rangle^{2}}-\frac{\left\langle n_{1}\right\rangle+\left\langle n_{2}\right\rangle+2\left\langle n_{1} n_{2}\right\rangle}{\left\langle n_{1}\right\rangle\left\langle n_{2}\right\rangle}
$$

\section{Results}

For the first time, experimental measurements of dynamical fluctuations of particle ratios are systematically confronted with theoretical predictions in Fig. 1. There was an earlier attempt to compare with preliminary results for $K / \pi$ reported in 3 . Is has been found that theoretical and experimental ratios of dynamical to statistical fluctuations are compatible with each other. Individual fluctuations themselves were not.

\footnotetext{
${ }^{1}$ Experimentally, there are various methods to construct statistical fluctuations [7. Frequently used method is the one that measures particle ratios from mixing events.
} 
Hadron resonance gas model is obviously able to reproduce all measurements. For $K^{+} / \pi^{+}$ratio, experimental data covers a wide range of square root of center of mass energy $\sqrt{s}$ [6] []. While for $(p+\bar{p}) /\left(\pi^{+}+\pi^{-}\right)$ratio, experimental data available so far has been measured at SPS energies only. That the results from hadron resonance gas model agree well with the existing experimental data, allows us to make predictions for other particle ratios as will be given in Fig. 2. On other hand, we can now use the model to systematically investigate dependence of event-by-event fluctuations on $\sqrt{s}$. Few comments are in order at this moment.

- Dependence of event-by-event fluctuations of particle ratios on $\sqrt{s}$ is non-monotonic

- Fluctuations can be suppressed and/or enhanced at different $\sqrt{s}$

- Strangeness fluctuations are positive and enhanced with $\sqrt{s}$. Anti-strangeness fluctuations are smaller than strangeness ones. There are remarkable minima at top SPS energies

- Non-strangeness fluctuations are negative, i.e., statistical fluctuations are greater than dynamical ones, especially at low energies.

- In general, event-by-event fluctuations at high energy increase with increasing energy. There is only one exception from this empirical role; $K^{-} / \pi^{-}$ratio. Fluctuations of $K^{-} / \pi^{-}$ratio exponentially decrease with $\sqrt{s}$.

In left panel of Fig. [1] we compare experimentally measured fluctuations of $K^{+} / \pi^{+}$ratios with resonance gas model results. There is a good agreement at SPS energies. At RHIC, measured fluctuations are slightly above the theoretical line. The explanation for this disagreement is twofolds. First, RHIC measurements are still preliminary [7. Second, we refer to our previous study of particle ratios in heavy ion collisions [13. We have concluded that thermal models with $\gamma=1$ slightly overestimate particle ratios at RHIC. To reproduce particle ratios, precisely, one has to allow $\gamma$ to take values different from unity ${ }^{2}$. Inserting large $\langle n\rangle$, averaged particle number density, in Eq. 8 apparently results in small $\sigma$.

In right panel in Fig. 1 dynamical fluctuations of $(p+\bar{p}) /\left(\pi^{+}+\pi^{-}\right)$ratios are depicted as a function of $\sqrt{s}$. According to Eq. 8 negative values of dynamical fluctuations are to be understood as a reason of much dominant statistical fluctuations. Nevertheless, using our model, we now have a quantitative estimation of both dynamical and statistical fluctuations. At low SPS energy, there is a good agreement between our model and the experimental results.

A much more important quantity is the ratio of dynamical fluctuations to statistical ones. This quantity will be given in Tab. 1at specific energies. Further advantage of right panel of Fig. 1is to illustrate the ability of our model. To author's knowledge, there is no another experimental data available.

Our predictions for dynamical fluctuations of different particle ratios are depicted in Fig. 2 Fluctuations of strangeness particle ratios are given in left panel. Right panel shows fluctuations of non-strangeness particle ratios. Depending on particle ratios, some fluctuations decrease and others increase with $\sqrt{s}$. As we discussed above, the energy dependence is apparently non-monotonic. Some particle ratios have negative dynamical fluctuations. Fluctuations of other particle ratios move from negative to positive values.

Negative fluctuations are explicitly obtained from non-strangeness particle ratios; dynamical fluctuations of $n / \pi^{-}, p / \pi^{+}$and $\pi^{+} / \pi^{-}$ratios are overall negative. Statistical fluctuations of such particle ratios are much greater than dynamical fluctuations. The fluctuations of $p / \pi^{-}$and $n / \pi^{+}$ have a remarkable dependence on $\sqrt{s}$. At low energy, i.e., AGS and SPS energies, they are negative. Within a relatively short energy interval, their values switch to positive sign. At higher energies, they monotonically raise with increasing $\sqrt{s}$.

The values of dynamical fluctuations of strangeness particle ratios are greater than that of nonstrangeness ones. Their dependence on $\sqrt{s}$ is also non-monotonic. While fluctuations from $K^{-} / \pi^{-}$ exponentially decrease with increasing $\sqrt{s}$, fluctuations from $\Lambda / \pi$ and $\Xi^{+} / \pi$ have minimum values at $\sqrt{s} \sim 10 \mathrm{GeV}$.

\footnotetext{
${ }^{2}$ Since available data is still preliminary, we can, for this moment, restrict our calculations to equilibrium value.
} 

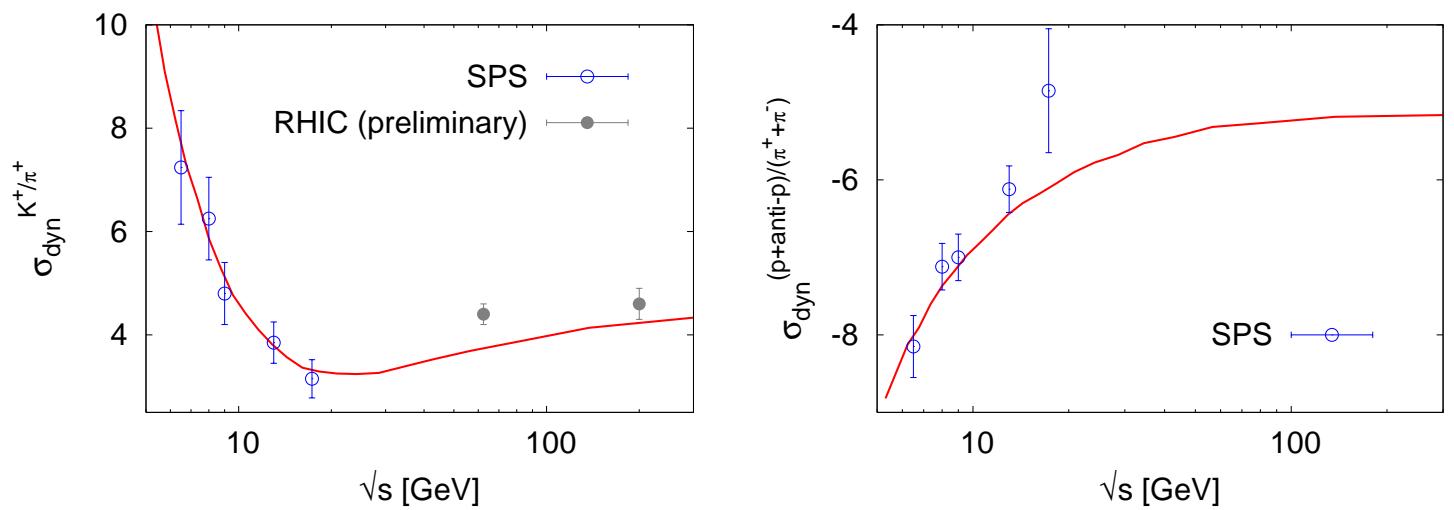

Fig. 1: Left panel: dynamical fluctuations of $K^{+} / \pi^{+}$ratio as a function of square root of center of mass energy (curve) compared with experimental results (circles). Results from SPS experiments [6] are drawn in open symbols. RHIC results are still preliminary (solid circles). Right panel shows dynamical fluctuations of $(p+\bar{p}) /\left(\pi^{+}+\pi^{-}\right)$ratios. Negative values are an indication to dynamical fluctuations that are smaller than statistical ones.
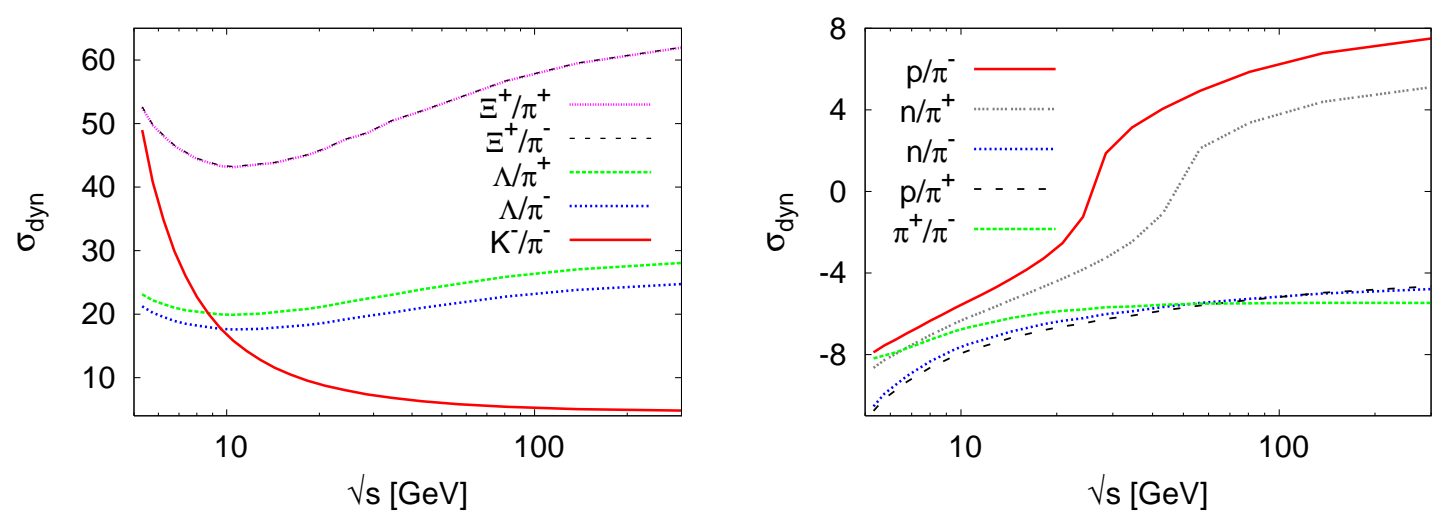

Fig. 2: Predictions for dynamical fluctuations of strangeness (left panel) and non-strangeness (right panel) particle ratios. Values of dynamical fluctuations at different energies strongly depend on particle ratios.

Comparing strangeness with non-strangeness fluctuations, we find that replacing pion by its anti-particle has almost no influence on strangeness dynamical fluctuations. Non-strangeness dynamical fluctuations are dramatically changed, when pion in denominator ${ }^{1}$ has been replaced by its anti-particle. For instance, for $p / \pi$ ratio

$$
\begin{aligned}
& \left\langle\left(\Delta n_{p / \pi^{+}}\right)^{2}\right\rangle \rightarrow\left\langle\left(\Delta n_{u}\right)^{2}\right\rangle+2\left\langle\left(\Delta n_{d}\right)^{2}\right\rangle \\
& \left\langle\left(\Delta n_{p / \pi^{-}}\right)^{2}\right\rangle \rightarrow 3\left\langle\left(\Delta n_{u}\right)^{2}\right\rangle
\end{aligned}
$$

As in right panel in Fig. 2] while fluctuations of first ratio move from negative to positive values, second ratio remains negative at all energies. In hadronic phase, i.e. particle ratios, quarks are strongly confined into hadronic states. It is believed that quarks in the unconfined phase may be strongly correlated 14. It would be interesting to verify above expressions. In doing this, we have to take into consideration volume fluctuations on lattice. Resonance gas model can not be applied at temperatures higher than critical one.

\footnotetext{
${ }^{1}$ Assuming that particle ratios can mathematically be seen as fractions.
} 


\section{Discussions and conclusions}

We find that the values of dynamical fluctuations depend on particle yields. According to QCD, hadronic matter under extreme conditions of high temperature and pressure is conjectured to have dynamical transitions into partonic matter. Assuming that we put certain particle ratio under these conditions, we want to see the change in its dynamical fluctuation before and after transition. This is equivalent to our numerical study at different energies. We start with hadronic matter of two particles and end with partonic mater, Eq. 9 and Eq. 10.

Fluctuations of quark number have been studied in lattice QCD [15]. It has been found that temperature-dependence (equivalent to $\sqrt{s}$-dependence) of fluctuations is dominated by analytic part of partition function. Across critical temperature, there is a smooth increase of fluctuations with increasing temperature. For our analysis, critical temperature is not exactly specified. Our results on fluctuations of individual particle are not shown here. Nevertheless, we can make following statements: We find that fluctuations of mesons (individual particles) have almost same structure as lattice fluctuations and that baryons have maximum value at $\sqrt{s} \approx 10 \mathrm{GeV}$. At higher energies, baryon fluctuations decrease. Quantitative comparison with lattice is - of course - not possible. As discussed in previous section, thermodynamics in hadronic phase can be reproduced when taking into account many stable and resonance hadrons in the partition function. A free gas of one hadron is not able to reproduce the quantitative thermodynamics below critical temperature.

In Tab凹 values of $\sigma_{d y n} / \sigma_{\text {stat }}$ quantity of different particle ratios are given at specific energies related to certain beam energies at top SPS and RHIC. It would - of course - be of great interest to verify these values experimentally. Our predictions specify the values of dynamical fluctuations related to statistical ones in all heavy ion collisions experiments. Experimentalists can now decide which particle ratios shall be measured. It depends - among others - on how large are dynamical fluctuations compared to statistical ones.

\begin{tabular}{|c||c|c|c|c|c|}
\hline & 12.3 & 17.3 & 62.4 & 100 & 200 \\
\hline \hline$K^{+} / \pi^{+}$ & 1.058 & 1.053 & 1.080 & 1.092 & 1.101 \\
\hline$K^{-} / \pi^{-}$ & 1.381 & 1.310 & 1.161 & 1.146 & 1.133 \\
\hline$\pi^{+} / \pi^{-}$ & 0.502 & 0.458 & 0.411 & 0.405 & 0.403 \\
\hline$(p+\bar{p}) /\left(\pi^{+}+\pi^{-}\right)$ & 0.607 & 0.675 & 0.803 & 0.814 & 0.821 \\
\hline$p / \pi^{-}$ & 0.851 & 0.927 & 1.107 & 1.139 & 1.163 \\
\hline$n / \pi^{+}$ & 0.778 & 0.848 & 1.028 & 1.059 & 1.081 \\
\hline$n / \pi^{-}$ & 0.605 & 0.676 & 0.847 & 0.873 & 0.897 \\
\hline$\Lambda / \pi^{+}$ & 1.501 & 1.531 & 1.573 & 1.575 & 1.576 \\
\hline$\Xi^{+} / \pi^{+}$ & 2.367 & 2.468 & 2.615 & 2.625 & 2.640 \\
\hline
\end{tabular}

Tab. 1: Ratios of $\sigma_{d y n} / \sigma_{\text {stat }}$ from different particle ratios at specific energies related to known beam energies at SPS and RHIC. With this quantity, we estimate how large are dynamical fluctuations compared to statistical ones at chemical freeze out in different heavy ion collisions experiments. Validity of these values depend on validity of chemical equilibrium freeze out scenario.

Strangeness fluctuation [16 are given in right panels of Fig. 1 and Fig. 2. The dependence of strangeness fluctuations on energy is also non-monotonic. For $K^{+} / \pi^{+}$ratios, fluctuations have a negative dependence on energy at SPS. There is a minimum around $\sqrt{s} \approx 20 \mathrm{GeV}$. At higher energies, fluctuations smoothly increase. Fluctuations of $\Lambda / \pi$ and $\Xi^{+} / \pi$ ratios have also minimum values at $\sqrt{s} \sim 10 \mathrm{GeV} . K^{-} / \pi^{-}$ratio has a completely different behavior. Its dynamical fluctuations of this particle ratio exponentially decrease with energy.

From our predictions for non-strangeness fluctuations drawn in right panel in Fig. 2] we find that event-by-event dynamical fluctuations depend on particle ratios. There is a rapid increase at low energy. At high energies, fluctuations smoothly increase. There is a remarkable sharp increase in $p / \pi^{-}$and $n / \pi^{+}$ratios within a short range of energies. The values of energies are different.

In final conclusion, we propose to study event-by-event dynamical fluctuations of different particle ratios in heavy ion collisions experiments. We have shown that baryon to meson fluctuations are much larger than meson to meson ones. Fluctuations of hybrid and cascade baryons are much 
larger than the statistical ones. According to our results, we now have a framework to study fluctuations of particle ratios, systematically. As mentioned above, fluctuations of particle ratios eliminates volume fluctuations. The latter are still included in lattice simulations.

We assumed that particle production is due to chemical equilibrium processes in final state, i.e., $\gamma=1$. That our models can very well reproduce experimental measurements means that the equilibrium freeze out scenario is apparently proved, especially at SPS energies. At RHIC, precise measurements are needed. Energy scan down to $\sqrt{s}=10 \mathrm{GeV}$ turns to be a crucial step to verify the worthwhile behavior of particle production [12, 13] and now dynamical fluctuations.

According to this systematic study, it was not possible to point out certain energy or region of energy, at which dynamical fluctuations sharply increase. The speculations on manifestation of critical endpoint by a rapidly increase of dynamical fluctuations of particle ratios can not be verified by this model. Furthermore, the region of unconfined phase can not be specified, precisely.

It would be interesting to extract information about the role of different decay channel in the energy-dependence of dynamical fluctuations. It will be a further propose to study the effect of chemical non-equilibrium processes of event-by-event dynamical fluctuations of particle ratios.

\section{Acknowledgment}

This work has been financially supported by the Japanese Society for the Promotion of Science.

\section{References}

[1] E. V. Shuryak, Phys. Lett. B 423:9, (1998)

[2] M. A. Stephanov, K. Rajagopal, E. V. Shuryak, Phys. Rev. D 60:114028 (1999)

[3] S. Jeon, V. Koch, Phys. Rev. Lett. 83:5435 (1999)

[4] G. Torrieri, S. Jeon, J. Rafelski, arXiv:nucl-th/0503026

[5] S. V. Afanasiev, et al. [NA49 Collaboration], Phys. Rev. Lett 86:1965 (2000)

[6] C. Rolland, et al. [NA49 Collaboration], J. Phys. G 30:S1381 (2004)

[7] S. Das [STAR Collaboration], arXiv:nucl-ex/0503023

[8] F. Karsch, K. Redlich, A. Tawfik, Eur. Phys. J. C 29:549, (2003)

[9] F. Karsch, K. Redlich, A. Tawfik, Phys. Lett. B 571:67, (2003)

[10] K. Redlich, F. Karsch, A. Tawfik, J. Phys., G 30:S1271, (2004)

[11] A. Tawfik, Phys. Rev. D 71:054502, (2005)

[12] A. Tawfik, J. Phys. G 31:S1105, (2005); arXiv:hep-ph/0410392 Nucl. Phys. A 764:387, (2006)

[13] A. Tawfik, arXiv:hep-ph/0505152 to appear in Prog. Theor. Phys.

[14] A. Tawfik, arXiv:hep-lat/0603018 arXiv:hep-ph/0604037

[15] S. Ejiri, F. Karsch, K. Redlich, Phys. Lett. B 633:275, (2006)

[16] M. Gazdzicki, arXiv:nucl-ex/0507017 